Bull. Austral. Math. Soc.

$28 \mathrm{D} 05,58 \mathrm{~F} 11,34 \mathrm{C} 11$

VOL. 58 (1998) [445-452]

\title{
TIME AVERAGES FOR CONTINUOUS FUNCTIONS ON DISTAL FLOWS
}

\author{
CARMEN NúNeZ
}

\begin{abstract}
We study the time averages of continuous functions along the trajectories of the distal projective flow induced by an ergodic family of Schrödinger equations. General conditions guaranteeing that the set of nonconvergence points is a residual subset are found. Applications to the study of the ergodic structure of the projective flow are given.
\end{abstract}

\section{INTRODUCTION}

This paper is concerned with the study of the time averages of continuous functions $h$ along the trajectories of a real flow $\mathbb{R} \times X \rightarrow X,(t, x) \mapsto x \cdot t$ on a compact metric space $X$; that is, we shall consider the set of $x$ 's such that the limit

$$
\lim _{T \rightarrow \infty} \frac{1}{2 T} \int_{-T}^{T} h(x \cdot t) d t
$$

exists, as well as the value of this limit. Johnson [5] shows that, when the flow on $X$ is minimal, then either the limit exists for all points of $X$ or the integral diverges for a residual subset of $X$. We intend to extend this result to flows with a more complicate topological structure: more precisely, to distal flows for which the set of minimal components has a simple geometrical structure.

Our model will be the flow induced on the real projective bundle $\Omega \times P^{1}(\mathbb{R})$ (where $\Omega$ is a compact metric space with a continuous flow and $P^{1}(\mathbb{R})$ denotes the real projective line) by a family of second-order linear Schrödinger equations, under the assumptions that the flow on $\Omega$ is minimal and all the solutions are bounded. This flow is distal, and the set of its minimal components (with the Hausdorff metric) can be identified with the unit circle (see below for details). The complete description of the ergodic and topological structures appears in Novo and Obaya [7] and Arnold, Cong and Oseledets [1], and it will allow us to prove that the set $K$ of nonconvergence points is residual in all the cases in which the union of the minimal components contained in $K_{\mathbb{R}}-K$ is a rare subset. This is the first purpose of the paper. The results will be used in the analysis of the set

Received 23rd March, 1998

Partially supported by Junta de Castilla y León under project VA01/97.

Copyright Clearance Centre, Inc. Serial-fee code: 0004-9729/98 \$A2.00+0.00. 
of definition and the continuity of a family of quadratic functions, which are associated with the perturbations of the potential and closely related to the ergodic and topological components of the projective flow.

Let us describe this flow more precisely. Let $\Omega$ be a compact metric space and $\Xi: \Omega \times \mathbb{R} \rightarrow \Omega,(\xi, t) \mapsto \xi \cdot t$ a continuous flow on $\Omega$. Given a real function $\Gamma_{0} \in C(\Omega)$ we consider the family of equations

$$
-x^{\prime \prime}+\Gamma_{0}(\xi \cdot t) x=0, \quad \xi \in \Omega
$$

Let $x(t, \xi, \varphi)$ be the solution of (1.1) along the trajectory of the point $\xi$ with initial data $x^{\prime}(0, \xi, \varphi)+\mathrm{i} x(0, \xi, \varphi)=\mathrm{e}^{\mathrm{i} \varphi}$. As usual, $P^{1}(\mathbb{R})$ and $P^{1}(\mathbb{C})$ denote the real and complex projective lines; the symbol $l_{1}$ stands for the normalised restriction of the Lebesgue measure of $\mathbb{R}$ to $P^{1}(\mathbb{R})$ (which we identify with the quotient space $\mathbb{R} / \pi \mathbb{Z}$ ), and $\Phi$ is the continuous skew-product flow induced by (1.1) on the projective bundles $K_{\mathbb{C}}=\Omega \times P^{1}(\mathbb{C})$ and $K_{\mathbb{R}}=\Omega \times P^{1}(\mathbb{R})$. We fix a $\Xi$-ergodic measure $m_{0}$ on $\Omega$ and define $m_{1}$ as the product measure $m_{0} \otimes l_{1}$ on a complete $\sigma$-algebra of $K_{\mathbb{R}}$.

Throughout this paper we shall assume that the flow $(\Omega, \Xi)$ is minimal and there exists $\xi_{0} \in \Omega$ such that the solutions of the corresponding equation (1.1) are bounded, which implies the boundedness of the solutions for every $\xi \in \Omega$. According to the results of [7], our assumptions also guarantee the existence of a $\Phi$-invariant measure on $K_{\mathbb{R}}$ which is absolutely continuous with respect to $m_{1}$ (see Obaya and Paramio [9] for the facts concerning the existence and methods of construction of this kind of measure). It is known that either this is the unique ergodic measure projecting onto $m_{0}$, or $K_{\mathbb{R}}$ decomposes into a family of $\Phi$-ergodic $k$-sheets, with $1 \leqslant k<\infty$. Therefore, when the projective flow is not uniquely ergodic, its ergodic components are ergodic $k$-sheets, which are, roughly speaking, $\Phi$-invariant measurable subsets of $K_{\mathbb{R}}$ (or $K_{\mathbb{C}}$ ) with the same number of points $(k)$ in the fibre for almost every $\xi \in \Omega$, and on which the $\Phi$-ergodic measures are concentrated (see also Furstenberg [4]).

Furthermore, as is shown in [7], $\left(K_{\mathbb{R}}, \Phi\right)$ admits an absolutely continuous invariant measure with continuous density function. The first consequence of this fact concerns the topological structure of the real projective bundle: the flow is distal, and hence either it is minimal or $K_{\mathbb{R}}$ decomposes into minimal subsets; more precisely, into closed ergodic $k$-sheets (see Ellis [3] and Sacker and Sell [10]). Whereas, in the first case, the minimal character of $K_{\mathbf{R}}$ does not preclude its decomposition into nonclosed ergodic $k$-sheets, in the second case the ergodic and topological structures of the projective bundle coincide (ergodic and minimal subsets are the same). Moreover, as we shall detail in Section 2, all the components are identical, and we can generate all of them from a single one in a continuous form. 
As stated before, the second purpose of the paper is the study of the limits

$$
q_{\Gamma}(\xi, \varphi)=\lim _{T \rightarrow \infty} \frac{1}{2 T} \int_{-T}^{T} \Gamma(\xi \cdot t) x^{2}(t, \xi, \varphi) d t
$$

for $\Gamma \in C(\Omega)$. Our hypotheses also guarantee that the limit exists for almost every $(\xi, \varphi) \in K_{\mathbb{R}}$ with respect to any $\Phi$-invariant measure and define a fibre-quadratic function

$$
q_{\Gamma}(\xi, \varphi)=a_{\Gamma}(\xi) \cos ^{2} \varphi+b_{\Gamma}(\xi) \sin ^{2} \varphi+2 c_{\Gamma}(\xi) \sin \varphi \cos \varphi
$$

The ergodic components of the projective bundles and the functions $q_{\Gamma}$ are closely related: the equation $q_{\Gamma}=0$ determines two conjugate complex 1-sheets (if $q_{\Gamma}>0$ or $q_{\Gamma}<0$ ), two real 1-sheets (if $q_{\Gamma}$ takes positive and negative values) or a unique real 1-sheet. Obviously, these sheets are closed (and hence minimal) when $q_{\Gamma}$ is continuous. Also, if $q_{\Gamma}>0$ or $q_{\Gamma}<0,1 /\left|q_{\Gamma}\right|$ is the density function of an absolutely continuous $\Phi$-invariant measure.

The functions $q_{\Gamma}$ are also fundamental in the study of the behaviour of the solutions of the perturbed equation $-x^{\prime \prime}+\Gamma_{0}(\xi \cdot t) x=E \Gamma(\xi \cdot t) x$ for small $E \in \mathbb{C}$. Núñez and Obaya [8] prove the nontangential $L^{1}$-convergence of the Weyl $m$-functions at $E=0$ for $\Gamma>0$ and obtain the limits from the expression of $q_{\Gamma}$. The boundedness of the solutions is not required. However, the result is stronger in the bounded orbit case: if $q_{\Gamma} \in C\left(K_{\mathbb{R}}\right)$ and is strictly positive or negative, then the perturbed equations admit exponential dichotomy for small nonreal $E$, the corresponding Weyl $m$-functions converge nontangentially at $E=0$, and the convergence is uniform (see [8] for details).

These facts point out the interest in analyzing the continuity of $q_{\Gamma}$. When the flow $(\Omega, \Xi)$ is uniquely ergodic, all the functions $q_{\Gamma}$ are continuous except in the case of decomposition of $K_{\mathbb{R}}$ into nonclosed ergodic 1-sheets. In the general case, if $K$ denotes the set of points of nonconvergence for (1.2), the function $q_{\Gamma}$ is continuous (or almost always equal to a continuous function) if and only if $K=\emptyset$, and in this case the limit (1.2) is uniform (see [8]). In this work we prove that if $K$ is not empty then it is a residual subset of $K_{\mathbb{R}}$. However, $\mu(K)=0$ for any $\Phi$-invariant measure $\mu$.

\section{Time aVERAges of CONTINUOUS FunCtions}

The main result of this paper (Theorem 2.3) is based on the following one, which generalises [5, Theorem 3.7]. An alternative and shorter proof is presented here.

THEOREM 2.1. Let $X$ be a complete metric space and $\Xi: \mathbb{R} \times X \rightarrow X,(t, x) \mapsto$ $x \cdot t$ a continuous flow on $X$. Suppose that $X$ is a minimal compact set or it decomposes into a family of minimal compact sets. Let $g \in C(X)$ be a function satisfying the following condition: for every minimal subset $M \subset X$ there exist two points $x_{1}, x_{2} \in M$ and a sequence of real numbers $\left(t_{n}\right)_{n \in \mathbf{N}}$ with $\lim _{n \rightarrow \infty} t_{n}=\infty$ such that

$$
\text { (h1) } \lim _{n \rightarrow \infty} \frac{1}{t_{n}} \int_{0}^{t_{n}} g\left(x_{1} \cdot s\right) d s=0
$$


(h2) the family $\left\{\int_{0}^{t} g\left(x_{2} \cdot s\right) d s \mid t \in \mathbb{R}\right\}$ is unbounded.

Then the set

$$
X_{1}=\left\{x \in X \mid \limsup _{t \rightarrow \infty} \int_{-t}^{t} g(x \cdot s) d s=\infty \text { and } \liminf _{t \rightarrow \infty} \int_{-t}^{t} g(x \cdot s) d s=-\infty\right\}
$$

is residual in $X$.

Proof: For each $T \geqslant 0$ we define

$$
d_{T}: X \longrightarrow[0,1], \quad x \mapsto \inf _{t \in[0, T]} \exp \left\{\int_{-t}^{t} g(x \cdot s) d s\right\} .
$$

Then $\left\{d_{T} \mid T \geqslant 0\right\}$ is a decreasing family of continuous functions, and hence the set $C$ of continuity points of $d=\lim _{T \rightarrow \infty} d_{T}$ is residual in $X$ (see Choquet [2]). From the definition of $d$ it is easy to check that for every $x \in X$ and $s \in \mathbb{R}$,

$$
d(x \cdot s) \leqslant \exp \left(2|s|\|g\|_{\infty}\right) d(x) .
$$

We prove that $C$ is contained in the $\Xi$-invariant set $\{x \in X \mid d(x)=0\}$. Assume, by way of contradiction, that $d\left(x_{0}\right)>0$ for a point $x_{0} \in C$. Then there exist an open neighbourhood $V$ of $x_{0}$ and a constant $\kappa_{1}>0$ such that $d(x)>\kappa_{1}$ for every $x \in V$. Let $M \subset X$ be the minimal set containing $x_{0}$. We can find $t_{1}, t_{2}, \ldots, t_{n}$ such that $M \subset V t_{1} \cup V t_{2} \cup \ldots \cup V t_{n}$. This property and (2.1) guarantee that $d(x) \geqslant \kappa_{1} \exp \left(-2 \bar{t}\|g\|_{\infty}\right)$ for every $x \in M$, where $\bar{t}=\max _{1 \leqslant i \leqslant n}\left|t_{i}\right|$. Therefore, there is $\kappa_{2} \in \mathbb{R}$ such that for every $x \in M$ and $t \geqslant 0, \int_{-t}^{t} g(x \cdot s) d s \geqslant-\kappa_{2}$, and, consequently,

$$
\int_{0}^{t} g(x \cdot s) d s=\int_{-t / 2}^{t / 2} g((x \cdot(t / 2)) \cdot s) d s \geqslant-\kappa_{2} .
$$

On the other hand, from hypothesis (h1) we deduce the existence of $x_{3} \in M$ such that the family $\left\{\int_{0}^{t} g\left(x_{3} \cdot s\right) d s \mid t \in \mathbb{R}\right\}$ is bounded (see [5, Lemma 3.5]). This fact, the minimal character of the flow on $M$ and (2.2) allow us to prove that $\left\{\int_{0}^{t} g(x \cdot s) d s \mid t \in \mathbb{R}\right\}$ is bounded for every $x \in M$, which contradicts condition (h2).

We conclude that there is a residual set of points in $X$ for which $d(x)=0$ or, equivalently, $\liminf _{t \rightarrow \infty} \int_{-t}^{t} g(x \cdot s) d s=-\infty$. The same arguments applied to $-g$ provide a new residual set on which $\limsup _{t \rightarrow \infty} \int_{-t}^{t} g(x \cdot s) d s=\infty$. The intersection of both sets, also residual in $X$, is the set $X_{1}$ of the statement.

Let $h$ be a continuous function on $K_{\mathrm{R}}$. As stated in the introduction, our aim is to study the $\Phi$-invariant set of points of convergence of the time averages,

$$
C_{h}=\left\{(\xi, \varphi) \in K_{\mathbf{R}} \mid \lim _{T \rightarrow \infty} \frac{1}{2 T} \int_{-T}^{T} h(\Phi(t, \xi, \varphi)) d t \text { exists }\right\} .
$$


Note that if a minimal set $M \subset K_{\mathrm{R}}$ is not contained in $C_{h}$, Johuson and Moser [6, Lemma 4.4] implies the existence of two $\Phi$-ergodic measures $\mu_{1}$ and $\mu_{2}$ concentrated on $M$ such that

$$
\int_{M} h(\xi, \varphi) d \mu_{1} \neq \int_{M} h(\xi, \varphi) d \mu_{2}
$$

This remark and an argument similar to the one of [5, Theorem 4.3] (combined with Theorem 2.1 above) lead us to the following result:

THEOREM 2.2. If $\left(K_{\mathbb{R}}, \Phi\right)$ is minimal and $h \in C\left(K_{\mathbb{R}}\right)$, then either $C_{h}=K_{\mathbb{R}}$ or the set $K_{\mathbb{R}}-C_{h}$ is residual in $K_{\mathbb{R}}$.

In order to establish the analogue when $\left(K_{\mathbb{R}}, \Phi\right)$ is not minimal, we recall several results from [7] concerning the minimal components of the real projective flow (which, as said before, are closed $\Phi$-ergodic $k$-sheets, with $k \geqslant 1$ ). Let us take a positive continuous density function $p$. According to [9], the function $p$ provides the homeomorphism

$$
H_{p}=\left(\operatorname{Id}, H_{p}^{2}\right): K_{\mathbb{R}} \rightarrow K_{\mathbb{R}}, \quad(\xi, \varphi) \mapsto\left(\xi, \int_{0}^{t} p(\xi \cdot s) d s\right)
$$

which takes the flow $\Phi$ to a skew-translation. Let $M$ be any one of the $k$-sheets and put $N=H_{p}(M)$. Then $N$ is an closed ergodic $k$-sheet for the transformed flow, and the $k$ elements of each fibre are equidistant (recall that $P^{\mathbf{l}}(\mathbb{R}) \equiv \mathbb{R} /(\pi \mathbb{Z})$ ). Besides, if for each $\delta \in[0, \pi / k)$ the set $N_{\delta}$ is defined by $\{(\xi, \psi+\delta) \mid(\xi, \psi) \in N\}$,

$$
K_{\mathbb{R}}=\bigcup_{\delta \in[0, \pi / k)} M_{\delta}, \quad \text { where } M_{\delta}=H_{p}^{-1}\left(N_{\delta}\right) .
$$

That is, the family of minimal components in which $K_{\mathbb{R}}$ decomposes is precisely $\mathcal{F}=\left\{M_{\delta}, \delta \in[0, \pi / k)\right\}$. Furthermore, if we consider the Hausdorff metric in $\mathcal{F}$, the map $[0, \pi / k) \rightarrow \mathcal{F}, \delta \mapsto M_{\delta}$ induces a homeomorphism between the quotient space $\mathbb{R} /((\pi / k) \mathbb{Z})$ and the metric space $\mathcal{F}$.

In particular, if $M_{\delta}$ and $M_{\bar{\delta}}$ are different $\Phi$-ergodic $k$-sheets, the map

$$
R_{\bar{\delta}-\delta}: K_{\mathbb{R}} \longrightarrow K_{\mathbb{R}}, \quad(\xi, \varphi) \mapsto H_{p}^{-1}\left(\xi, H_{p}^{2}(\xi, \varphi)+\bar{\delta}-\delta\right)
$$

is a homeomorphism which transforms $M_{\delta}$ into $M_{\bar{\delta}}$.

THEOREM 2.3. Assume that $K_{\mathbb{R}}$ decomposes into a collection of closed $\Phi$-ergodic $k$-sheets, and represent this family of components by $\left\{M_{\delta} \mid \delta \in[0, \pi / k)\right\}$ as in (2.5). Given a continuous function $h$ on $K_{\mathbf{R}}$, define $C_{h}$ by (2.3) and denote

$$
\Delta=\left\{\delta \in[0, \pi / k) \mid M_{\delta} \subset C_{h}\right\} \quad \text { and } \quad \widetilde{M}=\bigcup_{\delta \in \Delta} M_{\delta}=\bigcup_{M_{\delta} \subset C} M_{\delta} .
$$

Then, 
(i) if $\widetilde{M}$ is a rare set, then $K_{\mathbf{R}}-C_{h}$ is residual in $K_{\mathbf{R}}$;

(ii) the set $\widetilde{M}$ is rare in $K_{\mathbf{R}}$ if and only if $\Delta$ is rare in $[0, \pi / k)$.

Proof: Assume that $\widetilde{M}$ is a rare set. We fix $\delta \in[0, \pi / k)$ such that the $k$-sheet $M_{\delta}$ is not contained in $\widetilde{M}$; then there exist two $\Phi$-ergodic measures $\mu_{\delta, 1}$ and $\mu_{\delta, 2}$ concentrated on $M_{\delta}$ satisfying (2.4). For each $\bar{\delta} \in[0, \pi / k)$ we define two $\Phi$-invariant measures concentrated on $M_{\bar{\delta}}$ by

$$
\int_{M_{\bar{\delta}}} f(\xi, \varphi) d \nu_{\tilde{\delta}, i}=\int_{M_{\delta}} f \circ R_{\tilde{\delta}-\delta}(\xi, \varphi) d \mu_{\delta, i} \quad \text { for all } f \in C\left(K_{\mathbb{R}}\right), \quad i=1,2,
$$

where $R_{\bar{\delta}-\delta}$ is the homeomorphism given by (2.6), which transforms $M_{\delta}$ into $M_{\bar{\delta}}$. The functions

$$
h_{\delta, i}: K_{\mathbb{R}} \longrightarrow \mathbb{R}, \quad(\bar{\xi}, \bar{\varphi}) \mapsto \int_{M_{\bar{\delta}}} h(\xi, \varphi) d \nu_{\bar{\delta}, i} \quad \text { if }(\bar{\xi}, \bar{\varphi}) \in M_{\bar{\delta}}, \quad i=1,2
$$

are continuous, $\Phi$-invariant, and take constant values $h_{\bar{\delta}, i}^{*}$ on each $k$-sheet $M_{\bar{\delta}}$. Thus the set $B_{\delta}=\left\{(\xi, \varphi) \in K_{\mathbb{R}} \mid h_{\delta, 1}(\xi, \varphi) \neq h_{\delta, 2}(\xi, \varphi)\right\}$ is an open subset of $K_{\mathbb{R}}$ containing $M_{\delta}$. Note that $B_{\delta}=\bigcup_{h_{\tilde{\delta}, 1}^{*} \neq h_{\dot{\delta}, 2}^{\circ}} M_{\bar{\delta}}$, a union of $k$-sheets.

We prove that the set $C_{h} \cap B_{\delta}$ is of the first Baire category on $B_{\delta}$ (and hence on $\left.K_{\mathbb{R}}\right)$. Define $g_{\delta, 1}(\xi, \varphi)=h(\xi, \varphi)-h_{\delta, 1}(\xi, \varphi)$, which is a continuous function on $B_{\delta}$; then, for an arbitrary $k$-sheet $M_{\bar{\delta}} \subset B_{\delta}$,

$$
\int_{M_{\delta}} g_{\delta, 1}(\xi, \varphi) d \nu_{\bar{\delta}, 1}=0 \quad \text { and } \quad \int_{M_{\tilde{\delta}}} g_{\delta, 1}(\xi, \varphi) d \nu_{\delta, 2}=h_{\bar{\delta}, 2}^{*}-h_{\bar{\delta}, 1}^{*} \neq 0 .
$$

We deduce from the Birkhoff ergodic theorem that $g_{1}$ satisfies the hypotheses of Theorem 2.1. This provides a residual set $B_{1} \subset B_{\delta}$ such that for every $(\xi, \varphi) \in B_{1}$ there is a sequence $\left(t_{n}\right)_{n \in \mathbb{N}}$ of real numbers with $\lim _{n \rightarrow \infty} t_{n}=\infty$ satisfying $\int_{-t_{n}}^{t_{n}} g_{\delta, 1}(\Phi(s, \xi, \varphi)) d s=0$. The same argument applied to the function $g_{\delta, 2}(\xi, \varphi)=h(\xi, \varphi)-h_{\delta, 2}(\xi, \varphi)$ gives a residual set $B_{2} \subset B_{\delta}$ with the analogous property. Thus, for each $(\xi, \varphi) \in B_{1} \cap B_{2}$ we find two sequences $\left(t_{n}\right)_{n \in \mathbb{N}}$ and $\left(t_{n}^{*}\right)_{n \in \mathbb{N}}$ of real numbers with limit $\infty$ such that, if $(\xi, \varphi) \in M_{\bar{\delta}}$,

$$
\frac{1}{2 t_{n}} \int_{-t_{n}}^{t_{n}} h(\Phi(s, \xi, \varphi)) d s=h_{\delta_{, 1}^{*}}^{*} \quad \text { and } \quad \frac{1}{2 t_{n}^{*}} \int_{-t_{n}^{*}}^{t_{n}^{*}} h(\Phi(s, \xi, \varphi)) d s=h_{\delta, 2}^{*},
$$

and therefore the points of the residual set $B_{1} \cap B_{2}$ do not belong to $C_{h}$. That is, $C_{h} \cap B_{\delta}$ is of the first category: $C_{h} \cap B_{\delta}=\bigcup_{m \in \mathbb{N}} F_{\delta, m}$, where every $F_{\delta, m}$ is a rare set on $K_{\mathbb{R}}$.

For each $m \in \mathbb{N}$, the union of the $k$-sheets $M_{\delta}$ whose distance to $\widetilde{M}$ is greater than or equal to $1 / m$ is a $\Phi$-invariant compact set that we denote by $K_{m}$ (if $\widetilde{M}=\emptyset$ then $K_{m}=K_{\mathbb{R}}$ for every $\left.m \in \mathbb{N}\right)$. Since $K_{m} \subset \underset{M \in r K_{-}}{\bigcup} B_{\delta}$, it admits a finite subcovering. Note 
also that $K_{\mathbf{R}}-\widetilde{M}=\bigcup_{m \in \mathbb{N}} K_{m}$, so finally we find a countable family $\left\{\delta_{n} \mid n \in \mathbb{N}\right\}$ such that $K_{\mathbb{R}}-\widetilde{M} \subset \bigcup_{n \in \mathbb{N}} B_{\delta_{n}}$. We deduce that

$$
C_{h}=\widetilde{M} \bigcup\left(\bigcup_{n \in \mathbb{N}}\left(C_{h} \cap B_{\delta_{n}}\right)\right)=\widetilde{M} \bigcup\left(\bigcup_{n \in \mathbb{N}} \bigcup_{m \in \mathbb{N}} F_{\delta_{n}, m}\right)
$$

which is a countable union of rare sets. That is, $K_{\mathbb{R}}-C_{h}$ is residual in $K_{\mathbb{R}}$, and (i) is proved.

To check (ii), note that it suffices to show that the set $\bigcup_{\delta \in \Delta} N_{\delta}$ (with $N_{\delta}=H_{p}\left(M_{\delta}\right)$ ) is rare in $K_{\mathbb{R}}$ if and only if $\Delta$ is rare in $[0, \pi / k)$. We denote by $\bar{N}$ the closure of the set $N$, and by $\operatorname{int}(N)$ the set of its interior points. By considering the topological structure of $K_{\mathbb{R}}$ described above, is intuitively clear and not difficult to prove that

$$
\operatorname{int}\left(\overline{\bigcup_{\delta \in \Delta} N_{\delta}}\right)=\bigcup_{\delta \in \operatorname{int}(\bar{\Delta})} N_{\delta}
$$

which implies the result.

We can deduce from this Theorem the character of the set of definition of the limits $q_{\Gamma}(\xi, \varphi)$ for a continuous function $\Gamma$ on $\Omega$.

Corollary 2.4. Suppose that $\Gamma \in C(\Omega)$ and the limit $q_{\Gamma}(\xi, \varphi)$ given by (1.2) does not exist for every $(\xi, \varphi) \in K_{\mathbb{R}}$. Then there is a residual subset $K \subset K_{\mathbb{R}}$ of nonconvergence points.

Proof: Let us write $\Phi(t, \xi, \varphi)=(\xi \cdot t, \varphi(t, \xi, \varphi))$. The density function $p$ of a $\Phi$-invariant measure satisfies the relation

$$
p(\Phi(t, \xi, \varphi))=p(\xi, \varphi)\left|\left(x(t, \xi, \varphi), x^{\prime}(t, \xi, \varphi)\right)\right|^{2}
$$

and, besides,

$$
x(t, \xi, \varphi)=\sin \varphi(t, \xi, \varphi)\left|\left(x(t, \xi, \varphi), x^{\prime}(t, \xi, \varphi)\right)\right|
$$

(see [9]). We take a continuous positive density function $p$ and define $h(\xi, \varphi)=$ $\Gamma(\xi) \sin ^{2} \varphi p(\xi, \varphi)$, which is also continuous. Then, by (2.7) and (2.8),

$$
\lim _{T \rightarrow \infty} \frac{1}{2 T} \int_{-T}^{T} \Gamma(\xi \cdot t) x^{2}(t, \xi, \varphi) d t=\frac{1}{p(\xi, \varphi)} \lim _{T \rightarrow \infty} \frac{1}{2 T} \int_{-T}^{T} h(\Phi(t, \xi, \varphi)) d t .
$$

We denote by $C$ the set of convergence points for both limits. By hypothesis, $C \neq K_{\mathrm{z}}$.

Once again, the proof depends on the topological structure of the real projective flow. When $\left(K_{\mathbb{R}}, \Phi\right)$ is minimal, then the result follows from Theorem 2.2 . 
Suppose now that the minimal components are closed $\Phi$-ergodic $k$-sheets, with $k \geqslant 1$. It is easy to check that

$$
x(t, \xi, \varphi)=\frac{\sin \left(\varphi-\varphi_{2}\right)}{\sin \left(\varphi_{1}-\varphi_{2}\right)} x\left(t, \xi, \varphi_{1}\right)+\frac{\sin \left(\varphi_{1}-\varphi\right)}{\sin \left(\varphi_{1}-\varphi_{2}\right)} x\left(t, \xi, \varphi_{2}\right) .
$$

From this equality one can easily deduce that, given $\xi \in \Omega$, if the limits (1.2) exist for three distinct points $\left(\xi, \varphi_{1}\right),\left(\xi, \varphi_{2}\right)$ and $\left(\xi, \varphi_{3}\right)$, they exist for $(\xi, \varphi)$ for every $\varphi \in P^{1}(\mathbb{R})$ (see [8]). Consequently, if $k=1$ the set $C$ contains at most two different 1-sheets; when $k=2$ it contains at most a unique 2-sheet; and no $k$-sheet can be contained in $C$ in case $k \geqslant 3$. Under these conditions, we only have to apply Theorem 2.3 to complete the proof.

\section{REFERENCES}

[1] L. Arnold, Nguyen Dinh Cong and V. I. Oseledets, 'Jordan normal form for linear cocycles' (Report Nr. 360 Institut für Dynamische Systeme, Universität Bremen, 1997).

[2] G. Choquet, Lectures on Analysis, Vol. I (Benjamin, Massachusetts, 1969).

[3] R. Ellis, Lectures on Topological Dynamics (Benjamin, Massachusetts, 1969).

[4] H. Furstenberg, 'Strict ergodicity and transformations of the torus', Amer. J. Math. 85 (1961), 573-601.

[5] R. Johnson, 'Minimal functions with unbounded integral', Israel J. Math. 31 (1978), 133-141.

[6] R. Johnson and J. Moser, 'The rotation number for almost periodic differential equations', Commun. Math. Phys. 84 (1982), 403-438.

[7] S. Novo and R. Obaya, 'An ergodic classification of bidimensional linear systems', $J$. Dynamics Differential Equations 8 (1996), 373-406.

[8] C. Núñez and R. Obaya, 'Nontangential limit of the Weyl $m$-functions for the ergodic Schrödinger equation', J. Dynamics Differential Equations 10 (1998), 209-257.

[9] R. Obaya R. and M. Paramio, 'Directional differentiability of the rotation number for the almost periodic Schrödinger equation', Duke Math. J. 66 (1992), 521-552.

[10] R. J. Sacker and G. R. Sell, Lifting properties in skew-products flows with applications to differential equations, Mem. Amer. Math. Soc. 190 (Amer. Math. Soc., Providence, R.I., 1977).

Departamento de Matemática Aplicada a la Ingeniería

Universidad de Valladolid

Paseo del Cauce $\mathbf{s} / \mathbf{n}$

E-47011 Valladolid

Spain

e-mail: carnun@wmatem.eis.uva.es 